\title{
On two-dimensional superpotentials: from classical Hamilton-Jacobi theory to 2D supersymmetric quantum mechanics
}

\author{
A. Alonso Izquierdo ${ }^{(a)}$, M.A. Gonzalez Leon ${ }^{(a)}$ \\ M. de la Torre Mayado ${ }^{(b)}$ and J. Mateos Guilarte ${ }^{(b)}$ \\ (a) Departamento de Matematica Aplicada, Universidad de Salamanca, SPAIN \\ (b) Departamento de Fisica, Universidad de Salamanca, SPAIN
}

\begin{abstract}
Superpotentials in $\mathcal{N}=2$ supersymmetric classical mechanics are no more than the Hamilton characteristic function of the Hamilton-Jacobi theory for the associated purely bosonic dynamical system. Modulo a global sign, there are several superpotentials ruling Hamilton-Jacobi separable supersymmetric systems, with a number of degrees of freedom greater than one. Here, we explore how supersymmetry and separability are entangled in the quantum version of this kind of system. We also show that the planar anisotropic harmonic oscillator and the two-Newtonian centers of force problem admit two non-equivalent supersymmetric extensions with different ground states and Yukawa couplings.
\end{abstract}

\section{Introduction}

Supersymmetric quantum mechanics was tailor-designed for the purpose of studying the subtle and crucial concept of spontaneous supersymmetry breaking by E. Witten [1] in a context as basic and simple as possible. Very soon, the strength of that idea exploded in an unexpected direction: SUSY quantum mechanics on N-dimensional Riemannian manifolds [2] provided a physicist's approach to the very deep index theory of elliptic operators, with far reaching consequences for the exchange between the communities of mathematicians and physicists. The physics of supersymmetric quantum mechanics, however, was mainly studied in the case of only one degree of freedom. This task proved to be interesting enough to produce a huge body of literature; here we quote only References [3], [4], [5], and [6] as the background to our work.

Following previous work on the factorization method on $N$-dimensional quantum mechanical systems [7], the general formalism of multi-dimensional supersymmetric quantum mechanics was established in the mid-eighties by a Sankt-Petersburg group; see [8]. More recently, researchers in the entourage of the same group have explored the interplay between two-dimensional supersymmetric quantum mechanics with integrability and separability at the classical limit, [9], [11]. In Reference [12] we addressed this problem in a systematic way; we limited ourselves, however, to the classical theory as our scenario, profiting from the Hamilton-Jacobi equation to obtain the supersymmetric extension of classical invariants of Hamilton-Jacobi separable 2D systems. In the present work, our goal is to address the same issue in a purely quantum setting. We shall describe how the spectra of matrix differential operators of different rank are intertwined. We shall also show that the ground states (zero modes) have a particularly simple form in this kind of system.

The organization of the paper is as follows: in Section $\S 2$, for convenience of the reader, we summarize the general formalism of $\mathcal{N}=2$ supersymmetric quantum mechanics for systems with $N$ degrees of freedom. In order to set the stage for novel developments, we briefly rework the theoretical basis of N-dimensional SUSY quantum mechanics as originally presented in the papers [7]-[8]. They use the 
Clifford algebra formalism for the first time in this context, better than the exterior calculus of [1] for our purposes. We also try to adapt this framework to the cohomological approach proposed in Reference [13] to solve the supersymmetric Coulomb problem in any dimension by algebraic means. The entanglement between Hamilton-Jacobi theory and the separation of variables of the quantum Schrödinger equation is examined in Section $\S 3$. In Section $\S 4$ we discuss two interesting two-dimensional physical systems. Finally, we offer a brief Summary in Section $\S 5$.

\section{$2 \mathcal{N}=2$ supersymmetric quantum mechanics}

\section{$2.1 \quad N$-dimensional $\mathcal{N}=2$ SUSY quantum mechanics}

Let $\gamma^{j}, \gamma^{N+j}, j=1,2, \cdots, N$ be the Hermitian generators, $\left(\gamma^{j}\right)^{\dagger}=\gamma^{j},\left(\gamma^{N+j}\right)^{\dagger}=\gamma^{N+j}$, of the Clifford algebra $\mathbf{C}\left(\mathbf{R}^{2 N}\right)$ of $\mathbf{R}^{2 N}:\left\{\gamma^{j}, \gamma^{k}\right\}=2 \delta^{j k},\left\{\gamma^{N+j}, \gamma^{N+k}\right\}=2 \delta^{j k},\left\{\gamma^{j}, \gamma^{N+k}\right\}=0$, where $\{. .$.$\} denotes$ anticommutator. Because the dimension of the irreducible representation of $\mathbf{C}\left(\mathbf{R}^{2 N}\right)$ is $\sum_{f=0}^{N}\left(\begin{array}{c}N \\ f\end{array}\right)=2^{N}$, the generators of $\mathbf{C}\left(\mathbf{R}^{2 N}\right)$ are $2^{N} \times 2^{N}$ Hermitian matrices. The linear combinations $\psi_{+}^{j}=\frac{1}{2}\left(\gamma^{j}-\right.$ $\left.i \gamma^{N+j}\right), \psi_{-}^{j}=\frac{1}{2}\left(\gamma^{j}+i \gamma^{N+j}\right)$ of the generators satisfy the anticommutation rules: $\left\{\psi_{+}^{j}, \psi_{-}^{k}\right\}=\delta^{j k}$, $\left\{\psi_{+}^{j}, \psi_{+}^{k}\right\}=\left\{\psi_{-}^{j}, \psi_{-}^{k}\right\}=0$. Thus, $\psi_{+}^{j}$ and $\psi_{-}^{j}$ can be thought of as "creation" and "annihilation" fermionic operators. From these operators one defines the fermionic total number operator, $\hat{f}=\sum_{j=1}^{N} \psi_{+}^{j} \psi_{-}^{j}$, which allows one to assign a grading to the space of the irreducible representation of the Clifford algebra -the fermionic Fock space-: $\mathcal{F}=\mathcal{F}_{0} \oplus \mathcal{F}_{1} \oplus \cdots \oplus \mathcal{F}_{N}=\oplus_{f=0}^{N} \mathcal{F}_{f}, \hat{f} \mathcal{F}_{f}=f \mathcal{F}_{f}, \psi_{+}: \mathcal{F}_{f} \longrightarrow \mathcal{F}_{f+1}$, $\psi_{-}: \mathcal{F}_{f} \longrightarrow \mathcal{F}_{f-1}$.

The key ingredients in defining a $N$-dimensional quantum mechanical ${ }^{1}$ system with $\mathcal{N}=2$ supersymmetry are the supercharges:

$$
\begin{aligned}
& \hat{Q}_{+}=e^{W\left(x^{1}, \cdots, x^{N}\right)} \hat{Q}_{+}^{0} e^{-W\left(x^{1}, \cdots, x^{N}\right)}=i \sum_{j=1}^{N} \psi_{+}^{j}\left(\frac{\partial}{\partial x^{j}}-\frac{\partial W}{\partial x^{j}}\right) \quad, \quad \hat{Q}_{+}^{0}=i \sum_{j=1}^{N} \psi_{+}^{j} \frac{\partial}{\partial x^{j}} \\
& \hat{Q}_{-}=e^{-W\left(x^{1}, \cdots, x^{N}\right)} \hat{Q}_{-}^{0} e^{W\left(x^{1}, \cdots, x^{N}\right)}=i \sum_{j=1}^{N} \psi_{-}^{j}\left(\frac{\partial}{\partial x^{j}}+\frac{\partial W}{\partial x^{j}}\right) \quad, \quad \hat{Q}_{-}^{0}=i \sum_{j=1}^{N} \psi_{-}^{j} \frac{\partial}{\partial x^{j}}
\end{aligned}
$$

which change the number of fermions, $\hat{Q}_{+}: \mathcal{F}_{f} \longrightarrow \mathcal{F}_{f+1}, \hat{Q}_{-}: \mathcal{F}_{f} \longrightarrow \mathcal{F}_{f-1}$, and close the $\mathcal{N}=2$ SUSY algebra:

$$
\left\{\hat{Q}_{+}, \hat{Q}_{-}\right\}=2 \hat{H} \quad, \quad\left[\hat{Q}_{+}, \hat{H}\right]=\left[\hat{Q}_{-}, \hat{H}\right]=0 \quad, \quad \hat{Q}_{+}^{2}=0 \quad, \quad \hat{Q}_{-}^{2}=0 \quad .
$$

Here, $\hat{H}$ is the $\hat{Q}_{ \pm}$-invariant Hamiltonian:

$$
\hat{H}=-\frac{1}{2} \sum_{j=1}^{N}\left(\frac{\partial}{\partial x^{j}}+\frac{\partial W}{\partial x^{j}}\right)\left(\frac{\partial}{\partial x^{j}}-\frac{\partial W}{\partial x^{j}}\right) \mathbf{I}_{2^{N}}-\sum_{j=1}^{N} \sum_{k=1}^{N} \frac{\partial^{2} W}{\partial x^{j} \partial x^{k}} \psi_{+}^{j} \psi_{-}^{k} \quad .
$$

Compare these expressions with the Hamiltonians, supercharges and SUSY algebra of [8] (Section §3) and [13] (Section $\S 3$ ). The Hilbert space of states $\mathcal{H}=\mathcal{F} \otimes L^{2}\left(\mathbf{R}^{N}\right)$ inherits a grading from the fermionic Fock space:

$$
\mathcal{H}=\mathcal{H}_{0} \oplus \mathcal{H}_{1} \oplus \cdots \oplus \mathcal{H}_{N-1} \oplus \mathcal{H}_{N}=\oplus_{f=0}^{N} \mathcal{H}_{f} \quad, \quad \mathcal{H}_{f}=\mathcal{F}_{f} \otimes L^{2}\left(\mathbf{R}^{N}\right)
$$

Let us choose an orthonormal basis $\vec{e}_{j}, j=1,2, \cdot, N, \vec{e}_{j} \cdot \vec{e}_{k}=\delta_{j k}$ in $\mathbf{R}^{N}$. The Hamiltonian acting on $\mathcal{H}_{0}$ is an ordinary Schrödinger operator with potential energy:

$$
\hat{V}(\vec{x})=\frac{1}{2}\left(\vec{\nabla} W(\vec{x}) \vec{\nabla} W(\vec{x})+\nabla^{2} W(\vec{x})\right) \quad, \quad \vec{\nabla}=\sum_{j=1}^{N} \frac{\partial}{\partial x_{j}} \vec{e}_{j} \quad, \quad \nabla^{2}=\sum_{j=1}^{N} \frac{\partial^{2}}{\partial x_{j}^{2}}
$$

\footnotetext{
${ }^{1}$ We take a system of units where $\hbar=1$.
} 
i.e. it is obtained from the gradient and the Laplacian of the function $W$, called the superpotential for this reason. Acting on $\mathcal{H}_{f}$, however, $\hat{H}$ is a $\left(\begin{array}{c}N \\ f\end{array}\right) \times\left(\begin{array}{c}N \\ f\end{array}\right)$-matrix of differential operators but all the interactions are also determined by the superpotential $W$, see (4). In particular, the Yukawa terms -interactions sensitive to the fermionic number of the state- depend on the second partial derivatives of $W$. $W$ fully determines the supersymmetric mechanical system.

There is perfect analogy with de Rahm cohomology, see also [8] (Section $§ 4$ ). The SUSY charges play the rôle of the exterior derivative and its adjoint such that, in the SUSY complex,

$$
\mathcal{H}_{0} \underset{\hat{Q}_{-}}{\stackrel{\hat{Q}_{+}}{\rightleftarrows}} \mathcal{H}_{1} \underset{\hat{Q}_{-}}{\stackrel{\hat{Q}_{+}}{\rightleftarrows}} \mathcal{H}_{2} \underset{\hat{Q}_{-}}{\stackrel{\hat{Q}_{+}}{\rightleftarrows}} \cdots \cdots \underset{\hat{Q}_{-}}{\stackrel{\hat{Q}_{+}}{\rightleftarrows}} \mathcal{H}_{N-1} \underset{\hat{Q}_{-}}{\stackrel{\hat{Q}_{+}}{\rightleftarrows}} \mathcal{H}_{N}
$$

one defines the SUSY cohomology groups: $H^{f}(\mathcal{H}, \mathbf{C})=\frac{\operatorname{Ker} \hat{Q}_{+}^{f}}{\operatorname{Im} \hat{Q}_{+}^{f-1}} \quad$. Because the supercharges are nilpotent, there is a Hodge-type decomposition theorem - $\mathcal{H}=\hat{Q}_{+} \mathcal{H} \oplus \hat{Q}_{-} \mathcal{H} \oplus \operatorname{Ker} \hat{H}$ - where the kernel of $\hat{H}$ is a finite-dimensional subspace spanned by the zero modes. The proof is easy: invert $\hat{H}$ on the orthogonal subspace to $\operatorname{Ker} \hat{H}$ and write:

$$
\mathcal{H}^{\perp}=\frac{\hat{Q}_{+} \hat{Q}_{-}+\hat{Q}_{-} \hat{Q}_{+}}{\hat{H}} \mathcal{H}^{\perp}=\hat{Q}_{+}\left(\frac{\hat{Q}_{-}}{\hat{H}} \mathcal{H}^{\perp}\right)+\hat{Q}_{-}\left(\frac{\hat{Q}_{+}}{\hat{H}} \mathcal{H}^{\perp}\right)
$$

$\hat{H}$ plays the rôle of the Laplacian and we talk about $\hat{Q}_{ \pm}$-exact and $\hat{H}$-harmonic states.

As in Hodge theory, zero modes play a special rôle. $E=0$ eigenfunctions (zero modes) satisfy $\hat{Q}_{+} \Psi_{0}^{f}=\hat{Q}_{-} \Psi_{0}^{f}=0: \Psi_{0}^{f} \in \operatorname{Ker} \hat{H}$. If $\Psi_{0}^{f}=\hat{Q}_{+} \Phi_{0}^{f-1}, \hat{Q}_{-} \hat{Q}_{+} \Phi_{0}^{f-1}=0$ implies that $\left\|\Psi_{0}^{f}\right\|=\left\|\hat{Q}_{+} \Phi_{0}^{f-1}\right\|=$ 0 . Thus, non-trivial zero-energy states are all the $\hat{Q}_{ \pm}$-closed states that are not $\hat{Q}_{ \pm}$-exact. Spontaneous supersymmetry breaking will occur if all the cohomology groups $H^{f}(\mathcal{H}, \mathbf{C})$ are trivial. The Witten index is the Euler characteristic of the SUSY complex: $\operatorname{Tr}(-1)^{\hat{f}}=\sum_{f_{+}} \operatorname{dim} H^{f_{+}}(\mathcal{H}, \mathbf{C})-\sum_{f_{-}} \operatorname{dim} H^{f_{-}}(\mathcal{H}, \mathbf{C})$, where $f_{+}\left(f_{-}\right)$runs over even(odd) numbers of fermions; see [1]. This index is frequently used to decide whether or not a given system presents supersymmetry breaking because $\operatorname{Tr}(-1)^{\hat{f}}$ is easier to compute than the cohomology groups.

\subsection{Two-dimensional $\mathcal{N}=2$ SUSY quantum mechanics}

In systems with $N=2$ degrees of freedom, the formalism of $\mathcal{N}=2$ supersymmetric quantum mechanics can be developed quite explicitly. Creation and annihilation fermionic operators are defined from the four dimensional Dirac/Majorana matrices:

$$
\begin{gathered}
\psi_{+}^{1}=\frac{1}{2}\left(\gamma^{1}-i \gamma^{3}\right)=\left(\begin{array}{cccc}
0 & 0 & 0 & 0 \\
1 & 0 & 0 & 0 \\
0 & 0 & 0 & 0 \\
0 & 0 & 1 & 0
\end{array}\right) \\
\psi_{+}^{2}=\frac{1}{2}\left(\gamma^{2}-i \gamma^{4}\right)=\left(\begin{array}{cccc}
0 & 0 & 0 & 0 \\
0 & 0 & 0 & 0 \\
1 & 0 & 0 & 0 \\
0 & -1 & 0 & 0
\end{array}\right) \quad, \quad \psi_{-}^{1}=\frac{1}{2}\left(\gamma^{1}+i \gamma^{3}\right)=\left(\begin{array}{cccc}
0 & 1 & 0 & 0 \\
0 & 0 & 0 & 0 \\
0 & 0 & 0 & 1 \\
0 & 0 & 0 & 0
\end{array}\right)
\end{gathered}
$$

which are related to the operators $b_{1}^{\dagger}$ and $b_{2}^{\dagger}$ defined in [8] (Section $\S 5$ ). The supercharges are the $2 \times 2$-matrices of differential operators:

$$
\hat{Q}_{+}=i\left(\begin{array}{cccc}
0 & 0 & 0 & 0 \\
\frac{\partial}{\partial x^{1}}-\frac{\partial W}{\partial x^{1}} & 0 & 0 & 0 \\
\frac{\partial}{\partial x^{2}}-\frac{\partial W}{\partial x^{2}} & 0 & 0 & 0 \\
0 & -\frac{\partial}{\partial x^{2}}+\frac{\partial W}{\partial x^{2}} & \frac{\partial}{\partial x^{1}}-\frac{\partial W}{\partial x^{1}} & 0
\end{array}\right) ; \hat{Q}_{-}=i\left(\begin{array}{cccc}
0 & \frac{\partial}{\partial x^{1}}+\frac{\partial W}{\partial x^{1}} & \frac{\partial}{\partial x^{2}}+\frac{\partial W}{\partial x^{2}} & 0 \\
0 & 0 & 0 & -\frac{\partial}{\partial x^{2}}-\frac{\partial W}{\partial x^{2}} \\
0 & 0 & 0 & \frac{\partial}{\partial x^{1}}+\frac{\partial W^{1}}{\partial x^{1}} \\
0 & 0 & 0 & 0
\end{array}\right),
$$


which are nilpotent: $\hat{Q}_{+}^{2}=0=\hat{Q}_{-}^{2}$. The SUSY algebra

$$
\left\{\hat{Q}_{+}, \hat{Q}_{-}\right\}=2 \hat{H} \quad, \quad\left[\hat{Q}_{+}, \hat{H}\right]=\left[\hat{Q}_{-}, \hat{H}\right]=0
$$

closes in a Hamiltonian of the form

$$
2 \hat{H}=2\left(\begin{array}{ccc}
\hat{h}^{(0)} & 0 & 0 \\
0 & \hat{h}^{(1)} & 0 \\
0 & 0 & \hat{h}^{(2)}
\end{array}\right)
$$

where

$$
2 \hat{h}^{(f=0)}=-\nabla^{2}+\vec{\nabla} W \vec{\nabla} W+\nabla^{2} W \quad \text { and } \quad 2 \hat{h}^{(f=2)}=-\nabla^{2}+\vec{\nabla} W \vec{\nabla} W-\nabla^{2} W \quad,
$$

are ordinary Schrödinger operators, and

$$
2 \hat{h}^{(f=1)}=\left(\begin{array}{cc}
-\nabla^{2}+\vec{\nabla} W \vec{\nabla} W-\square^{2} W & -2 \frac{\partial^{2} W}{\partial x^{1} \partial x^{2}} \\
-2 \frac{\partial^{2} W}{\partial x^{1} \partial x^{2}} & -\nabla^{2}+\vec{\nabla} W \vec{\nabla} W+\square^{2} W
\end{array}\right), \square^{2}=\frac{\partial^{2}}{\partial x^{1} \partial x^{1}}-\frac{\partial^{2}}{\partial x^{2} \partial x^{2}}
$$

is a $2 \times 2$ - matrix Schrödinger operator; see [8], Section $\S 5$.

Given an eigenstate of $\hat{H}$ in $\mathcal{H}_{0}$ with $E \neq 0$,

$$
\hat{h}^{(0)} \psi_{E}\left(x^{1}, x^{2}\right)=E \psi_{E}\left(x^{1}, x^{2}\right) \quad, \quad \Psi_{E}^{(0)}\left(x^{1}, x^{2}\right)=\left(\begin{array}{c}
\psi_{E}\left(x^{1}, x^{2}\right) \\
0 \\
0 \\
0
\end{array}\right),
$$

we have that $\hat{Q}_{-} \Psi_{E}^{(0)}\left(x^{1}, x^{2}\right)=0$-it is $\hat{Q}_{- \text {-closed-. However, }}$

$$
\hat{Q}_{+} \Psi_{E}^{(0)}\left(x^{1}, x^{2}\right)=i\left(\begin{array}{c}
0 \\
\left(\frac{\partial}{\partial x^{1}}-\frac{\partial W}{\partial x^{1}}\right) \psi_{E}\left(x^{1}, x^{2}\right) \\
\left(\frac{\partial}{\partial x^{2}}-\frac{\partial W}{\partial x^{2}}\right) \psi_{E}\left(x^{1}, x^{2}\right) \\
0
\end{array}\right)
$$

is a eigenstate of $\hat{H}$ with the same energy and fermionic number $f=1$ :

$$
\hat{h}^{(1)}\left(\begin{array}{c}
\left(\frac{\partial}{\partial x^{1}}-\frac{\partial W}{\partial x^{1}}\right) \psi_{E}\left(x^{1}, x^{2}\right) \\
\left(\frac{\partial}{\partial x^{2}}-\frac{\partial W}{\partial x^{2}}\right) \psi_{E}\left(x^{1}, x^{2}\right)
\end{array}\right)=E\left(\begin{array}{c}
\left(\frac{\partial}{\partial x^{1}}-\frac{\partial W}{\partial x^{1}}\right) \psi_{E}\left(x^{1}, x^{2}\right) \\
\left(\frac{\partial}{\partial x^{2}}-\frac{\partial W}{\partial x^{2}}\right) \psi_{E}\left(x^{1}, x^{2}\right)
\end{array}\right) \quad ;
$$

$\hat{h}^{(0)}$ is intertwined with $\hat{h}^{(1)}$ and one says that $\Psi_{E}^{(1)}=\hat{Q}_{+} \Psi_{E}^{(0)}$ is a $\hat{Q}_{+}$-exact state. Simili modo, starting from eigenstates of $\hat{H}$ with $E \neq 0$ in $\mathcal{H}_{2}$-all of them $\hat{Q}_{+}$-closed, i.e. $\hat{Q}_{+} \Psi_{E}^{(2)}=0$ - ,

$$
\hat{h}^{(2)} \phi_{E}\left(x^{1}, x^{2}\right)=E \phi_{E}\left(x^{1}, x^{2}\right) \quad, \quad \Psi_{E}^{(2)}\left(x^{1}, x^{2}\right)=\left(\begin{array}{c}
0 \\
0 \\
0 \\
\phi_{E}\left(x^{1}, x^{2}\right)
\end{array}\right),
$$

one easily sees that - the $\hat{Q}_{-}$-exact state-

$$
\hat{Q}_{-} \Psi_{E}^{(2)}\left(x^{1}, x^{2}\right)=i\left(\begin{array}{c}
0 \\
-\left(\frac{\partial}{\partial x^{2}}+\frac{\partial W}{\partial x^{2}}\right) \phi_{E}\left(x^{1}, x^{2}\right) \\
\left(\frac{\partial}{\partial x^{1}}+\frac{\partial W}{\partial x^{1}}\right) \phi_{E}\left(x^{1}, x^{2}\right) \\
0
\end{array}\right)
$$

is a eigenstate of $\hat{H}$ :

$$
\hat{h}^{(1)}\left(\begin{array}{c}
\left(-\frac{\partial}{\partial x^{2}}-\frac{\partial W}{\partial x^{2}}\right) \phi_{E}\left(x^{1}, x^{2}\right) \\
\left(\frac{\partial}{\partial x^{1}}+\frac{\partial W}{\partial x^{1}}\right) \phi_{E}\left(x^{1}, x^{2}\right)
\end{array}\right)=E\left(\begin{array}{c}
\left(-\frac{\partial}{\partial x^{2}}-\frac{\partial W}{\partial x^{2}}\right) \phi_{E}\left(x^{1}, x^{2}\right) \\
\left(\frac{\partial}{\partial x^{1}}+\frac{\partial W}{\partial x^{1}}\right) \phi_{E}\left(x^{1}, x^{2}\right)
\end{array}\right)
$$

$\hat{h}^{(2)}$ and $\hat{h}^{(1)}$ are also intertwined. Note, however, that $\left\langle\hat{Q}_{-} \Psi_{E}^{(2)} \mid \hat{Q}_{+} \Psi_{E}^{(0)}\right\rangle=0$ and $\hat{h}^{(0)}$ is not intertwined with $\hat{h}^{(2)}$. See Reference [10] to find how two scalar Hamiltonians are intertwined through second-order supercharges. 


\subsection{Zero energy eigenstates: spontaneous symmetry breaking}

The zero energy wave functions for the scalar Hamiltonians satisfy respectively: $\hat{Q}_{+} \Psi_{0}^{(0)}\left(x^{1}, x^{2}\right)=0$, $\hat{Q}_{-} \Psi_{0}^{(2)}\left(x^{1}, x^{2}\right)=0$. Therefore, $\vec{\nabla} \log \psi_{0}\left(x^{1}, x^{2}\right)=\vec{\nabla} W, \vec{\nabla} \log \phi_{0}\left(x^{1}, x^{2}\right)=-\vec{\nabla} W$, and

$$
\Psi_{0}^{(0)}\left(x^{1}, x^{2}\right)=C\left(\begin{array}{c}
\exp \left[W\left(x^{1}, x^{2}\right)\right] \\
0 \\
0 \\
0
\end{array}\right) \quad, \quad \Psi_{0}^{(2)}\left(x^{1}, x^{2}\right)=C\left(\begin{array}{c}
0 \\
0 \\
0 \\
\exp \left[-W\left(x^{1}, x^{2}\right)\right]
\end{array}\right) .
$$

There are normalizable zero-energy states in $\mathcal{H}_{0}$ or $\mathcal{H}_{2}$ - and $H^{f=0}(\mathcal{H}, \mathbf{C})$ or $H^{f=2}(\mathcal{H}, \mathbf{C})$ are non-trivialif

$$
\iint_{\mathbb{R}^{2}} d x^{1} d x^{2} e^{2 W\left(x^{1}, x^{2}\right)}<+\infty \quad \text { or } \quad \iint_{\mathbb{R}^{2}} d x^{1} d x^{2} e^{-2 W\left(x^{1}, x^{2}\right)}<+\infty \quad .
$$

Unbroken supersymmetry due to bosonic zero modes arise in 2D SUSY quantum mechanics under the same requirements as in 1D SUSY quantum mechanics, see [15]. However, the search for wave functions belonging to $\operatorname{Ker} \hat{h}^{(1)}$ is slightly more difficult.

$$
\hat{Q}_{-} \Psi_{0}^{(1)}\left(x^{1}, x^{2}\right)=0=\hat{Q}_{+} \Psi_{0}^{(1)}\left(x^{1}, x^{2}\right)
$$

requires integration of the equations

$$
\vec{\nabla} \log \xi_{0}\left(x^{1}, x^{2}\right)=-\frac{\partial W}{\partial x^{1}} \vec{e}_{1}+\frac{\partial W}{\partial x^{2}} \vec{e}_{2} \quad, \quad \vec{\nabla} \log \eta_{0}\left(x^{1}, x^{2}\right)=\frac{\partial W}{\partial x^{1}} \vec{e}_{1}-\frac{\partial W}{\partial x^{2}} \vec{e}_{2} .
$$

Note that in the odd cases the gradient of the log of the wave function is equal to the gradient of the superpotential on a plane with the reverse orientation. The solutions of (9) are:

$\Psi_{0}^{(1)}\left(x^{1}, x^{2}\right)=C_{1}\left(\begin{array}{c}0 \\ \exp \left[\tilde{W}\left(x^{1}, x^{2}\right)\right] \\ 0 \\ 0\end{array}\right)+C_{2}\left(\begin{array}{c}0 \\ 0 \\ \exp \left[-\tilde{W}\left(x^{1}, x^{2}\right)\right] \\ 0\end{array}\right)=C_{1}\left(\begin{array}{c}0 \\ \xi_{0}\left(x^{1}, x^{2}\right) \\ 0 \\ 0\end{array}\right)+C_{2}\left(\begin{array}{c}0 \\ 0 \\ \eta_{0}\left(x^{1}, x^{2}\right) \\ 0\end{array}\right)$,

where $\tilde{W}$ is such that: $\frac{\partial \tilde{W}}{\partial x^{1}}=-\frac{\partial W}{\partial x^{1}}, \frac{\partial \tilde{W}}{\partial x^{2}}=\frac{\partial W}{\partial x^{2}}$. There are normalizable zero-energy states in $\mathcal{H}_{1}-$ and $H^{f=1}(\mathcal{H}, \mathbf{C})$ is non-trivial- if either

$$
\iint_{\mathbb{R}^{2}} d x^{1} d x^{2} e^{2 \tilde{W}\left(x^{1}, x^{2}\right)}<+\infty \quad \text { and/or } \quad \iint_{\mathbb{R}^{2}} d x^{1} d x^{2} e^{-2 \tilde{W}\left(x^{1}, x^{2}\right)}<+\infty \quad .
$$

There are requirements on the superpotential to find unbroken supersymmetry coming from fermionic zero modes similar to those met in the bosonic sectors.

\section{Hamilton-Jacobi theory, supersymmetry and separability}

The quantum system described in Section $\S 2$ enjoys $\mathcal{N}=2$ supersymmetry by construction; the datum needed to set the interactions is the superpotential $W(\vec{x})$. Alternatively, there might be interest in knowing if a given Hamiltonian admits $\mathcal{N}=2$ supersymmetry; in that case, the datum is the potential energy $\hat{V}(\vec{x})$ and the identification of the superpotential requires that the Riccati-like PDE (5) must be solved. In [13], the superpotential for the quantum Coulomb problem is shown to be: $W\left(x_{1}, x_{2}\right)=$ $\sqrt{2 \lambda} \sqrt{x_{1}^{2}+x_{2}^{2}}$. Temporarily recovering the Planck constant, one finds:

$$
\frac{1}{2} \vec{\nabla} W \vec{\nabla} W=\lambda \quad ; \quad \frac{1}{2}\left(\vec{\nabla} W \vec{\nabla} W \pm \hbar \nabla^{2} W\right)=\lambda\left[1 \pm \frac{\hbar}{2} \sqrt{\frac{2}{\lambda}} \cdot \frac{1}{r}\right] \quad .
$$


The classical and zero-Grasmann limit of this supersymmetric system is therefore the free particle; the second partial derivatives of the superpotential arising in $\hat{h}^{(1)}$ are also multiplied by $\hbar$.

In [14], the superpotential for the supersymmetric Coulomb problem is chosen in such a way that the Coulomb potential energy arises at the classical non-Grassman limit: $W\left(x_{1}, x_{2}\right)=2 \sqrt{2 \lambda}\left(x_{1}^{2}+x_{2}^{2}\right)^{\frac{1}{4}}$ is the solution of the Hamilton-Jacobi equation for the Coulomb problem, instead of (5):

$$
\frac{1}{2} \vec{\nabla} W \vec{\nabla} W=\frac{\lambda}{r} \quad ; \quad \frac{1}{2}\left(\vec{\nabla} W \vec{\nabla} W \pm \hbar \nabla^{2} W\right)=\frac{\lambda}{r}\left[1 \pm \frac{\hbar}{4} \sqrt{\frac{2}{\lambda}} \cdot \frac{1}{r^{\frac{1}{2}}}\right] \quad .
$$

We shall follow this point of view and briefly summarize the connection between the superpotential and the solutions of the Hamilton-Jacobi equation, an issue fully developed in Reference [12]. Interesting work on the link between 2D classical integrable systems and SUSY quantum mechanics has also been performed in [9]. We stress, however, that it is not equivalent first to solve the HJ equation, define the classical supercharges, and, then to quantize these latter as to first quantize the purely bosonic system, solve (5), and then define the quantum supercharges.

\subsection{Hamiltonian formalism and the Hamilton characteristic function}

Let the $\mathcal{N}=2$ classical SUSY Hamiltonian be:

$$
H=\frac{1}{2} \sum_{j=1}^{N} p_{j} p_{j}+\frac{1}{2} \sum_{j=1}^{N} \frac{\partial W}{\partial x^{j}} \frac{\partial W}{\partial x^{j}}-i \sum_{j=1}^{N} \sum_{k=1}^{N} W_{j k} \theta_{2}^{j} \theta_{1}^{k} \quad, \quad W_{j k}=\frac{\partial^{2} W}{\partial x^{j} \partial x^{k}}
$$

The momenta and coordinates in the phase superspace are $p_{j}, x^{j}, \theta_{1}^{j}, \theta_{2}^{j}$, where $\theta_{1}^{j}$ and $\theta_{2}^{j}$ are the up and down components of $N$ Grassman Majorana spinors: $\left(\begin{array}{c}\theta_{1}^{j} \\ \theta_{2}^{j}\end{array}\right), \theta_{\alpha}^{j} \theta_{\beta}^{k}+\theta_{\beta}^{k} \theta_{\alpha}^{j}=0, \alpha, \beta=1,2$.

The Poisson superbrackets of any superfunction on the superspace $\{F, G\}_{P}=\frac{\partial F}{\partial p_{j}} \frac{\partial G}{\partial x^{j}}-\frac{\partial F}{\partial x^{j}} \frac{\partial G}{\partial p_{j}}+$ $i F \frac{\overleftarrow{\partial}}{\partial \theta_{\alpha}^{j}} \frac{\vec{\partial}}{\partial \theta_{\alpha}^{j}} G$ are obtained from the Poisson superstructure defined by the basic superbrackets:

$$
\left\{p_{j}, x^{k}\right\}_{P}=\delta_{j}^{k} \quad\left\{x^{j}, x^{k}\right\}_{P}=\left\{p_{j}, p_{k}\right\}_{P}=0 \quad\left\{\theta_{\alpha}^{j}, \theta_{\beta}^{k}\right\}_{P}=i \delta^{j k} \delta_{\alpha \beta} \quad .
$$

The classical SUSY charges

$$
Q_{1}=\sum_{j=1}^{N}\left(p_{j} \theta_{1}^{j}-\frac{\partial W}{\partial x^{j}} \theta_{2}^{j}\right) \quad, \quad Q_{2}=\sum_{j=1}^{N}\left(p_{j} \theta_{2}^{j}+\frac{\partial W}{\partial x^{j}} \theta_{1}^{j}\right)
$$

close the classical SUSY algebra: $\left\{Q_{1}, Q_{1}\right\}_{P}=\left\{Q_{2}, Q_{2}\right\}_{P}=2 i H,\left\{Q_{\alpha}, H\right\}_{P}=0,\left\{Q_{1}, Q_{2}\right\}_{P}=-i p_{j} \frac{\partial W}{\partial x^{j}}$.

In the canonical quantization procedure. Poisson superbrackets are promoted to supercommutators: $\left[\hat{x}^{j}, \hat{p}^{k}\right]=i \delta^{j k},\left\{\hat{\theta}_{\alpha}^{j}, \hat{\theta}_{\beta}^{k}\right\}=-\delta^{j k} \delta_{\alpha \beta}$. The representation of this Heisenberg superalgebra by $\hat{p}^{j}=\frac{1}{i} \frac{\partial}{\partial x^{j}}, \hat{x}^{j}=x^{j}, \hat{\theta}_{1}^{j}=\psi_{1}^{j}, \hat{\theta}_{2}^{j}=\psi_{2}^{j}$, where $\psi_{1}^{j}=\frac{i}{\sqrt{2}}\left(\psi_{+}^{j}+\psi_{-}^{j}\right)=\frac{i}{\sqrt{2}} \gamma^{j}, \psi_{2}^{j}=\frac{1}{\sqrt{2}}\left(\psi_{+}^{j}-\psi_{-}^{j}\right)=-\frac{i}{\sqrt{2}} \gamma^{N+j}$ are the Majorana $\gamma$-matrices, leads to the quantum supercharges (1), (2) and the quantum superalgebra (3) of Section $\S 2$.

Setting all the Grassman variables $\theta_{\alpha}^{j}$ equal to zero -the "body" of the superspace- , we have a Hamiltonian dynamical system with Hamiltonian and Hamilton-Jacobi equation:

$$
H=\frac{1}{2} \sum_{j=1}^{N} p_{j} p_{j}+V\left(x^{1}, x^{2}, \cdots, x^{N}\right) \quad, \quad \frac{\partial S}{\partial t}+H\left(\frac{\partial S}{\partial x^{1}}, \frac{\partial S}{\partial x^{2}}, \cdots, \frac{\partial S}{\partial x^{n}}, x^{1}, x^{2}, \cdots, x^{N}\right)=0
$$


There being no explicit dependence on time in $H$, one looks for solutions of the form $S\left(t, x^{1}, x^{2}, \cdots, x^{N}\right)=$ $W\left(x^{1}, x^{2}, \cdots, x^{N}\right)-i_{1} t$, and the time-independent Hamilton-Jacobi equation reads:

$$
i_{1}=\frac{1}{2} \sum_{j=1}^{N} \frac{\partial W}{\partial x^{j}} \frac{\partial W}{\partial x^{j}}+V\left(x^{1}, x^{2}, \cdots, x^{N}\right)
$$

$W\left(x^{1}, x^{2}, \cdots, x^{N}\right)$ is usually referred to as the Hamilton characteristic function. Assuming semi-definite positive potential energy $-U\left(x^{1}, x^{2}, \cdots, x^{N}\right) \geq 0$-, we state the following:

The superpotential of a $N$-dimensional $\mathcal{N}=2$ supersymmetric dynamical system is a solution of the time - independent Hamilton - Jacobi equation (11) for $i_{1}=0$ and $V(\vec{x})=-U(\vec{x})$.

Therefore, there are as many superpotentials as there are solutions of the Hamilton-Jacobi equation with zero energy in minus the potential energy of the body of the supersymmetric system. More precisely: given a Hamiltonian system with potential energy $U\left(x^{1}, x^{2}, \cdots, x^{N}\right)$, there are as many $\mathcal{N}=2$ supersymmetric extensions as there are zero-energy solutions of the Hamilton-Jacobi equation (11) for $V\left(x^{1}, x^{2}, \cdots, x^{N}\right)=-U\left(x^{1}, x^{2}, \cdots, x^{N}\right)$.

Further understanding of the consequences of this statement is provided by systems for which the Hamilton-Jacobi equation is separable. Separability in connection with pseudo-Hermitcity has been considered in the context of 2D SUSY quantum mechanics in [11]. In particular, if $U\left(x^{1}, x^{2}, \cdots, x^{N}\right)=$ $\sum_{j=1}^{N} U_{j}\left(x^{j}\right)$, there are $2^{N}$ solutions of (11). If there are no cyclic coordinates,

$$
W^{\left(a_{1}, a_{2}, \cdots, a_{N}\right)}\left(x^{1}, x^{2}, \cdots, x^{N}\right)=(-1)^{a_{1}} W_{1}\left(x^{1}\right)+(-1)^{a_{2}} W_{2}\left(x^{2}\right)+\cdots+(-1)^{a_{N}} W_{N}\left(x^{N}\right) \quad,
$$

where $a_{1}, a_{2}, \cdots, a_{N}=0,1 . \quad N=2$-dimensional systems for which the Hamilton-Jacobi equation is separable in Cartesian coordinates are called Type IV Liouville systems; see [16]. In this case, changing a global sign in $W^{(0,0)}$ merely exchanges $\hat{h}^{0}$ by $\hat{h}^{2}$ and $\hat{h}_{11}^{1}$ by $\hat{h}_{22}^{1}$ : i.e., it is tantamount to Hodge duality. Choosing $W^{(0,1)}\left(x^{1}, x^{2}\right)=W_{1}\left(x^{1}\right)-W_{2}\left(x^{2}\right)$ instead of $W^{(0,0)}\left(x^{1}, x^{2}\right)=W_{1}\left(x^{1}\right)+W_{2}\left(x^{2}\right)$, one replaces $W$ by $\tilde{W}$ and the second supersymmetric extension based on $\tilde{W}$ exhibits a fermionic zero mode if the first extension has a bosonic zero mode. The other eigenfunctions also change and the supersymmetric systems are not equivalent.

Even if the Hamilton-Jacobi equation is not separable, one can still envisage situations where a manifold of solutions is available. Let us consider a Hamiltonian system with two degrees of freedom and potential energy:

$$
U\left(x^{1}, x^{2}\right)=\lambda^{2}\left(x^{1} x^{1}+x^{2} x^{2}\right)^{n}-2 \lambda \alpha\left(x^{1} x^{1}+x^{2} x^{2}\right)^{\frac{n}{2}} \cos \left[n \arctan \left\{\frac{x^{2}}{x^{1}}\right\}\right]+\alpha^{2} \quad,
$$

where $\lambda$ and $\alpha$ are real physical parameters. It is not difficult to show, see [17], that there is a circle of zero energy solutions of the Hamilton-Jacobi equation with $V\left(x^{1}, x^{2}\right)=-U\left(x^{1}, x^{2}\right)$. If we define

$$
\begin{aligned}
& W\left(x^{1}, x^{2}\right)=\frac{\lambda}{n}\left(x^{1} x^{1}+x^{2} x^{2}\right)^{\frac{n}{2}} \cos \left[n \arctan \left(\frac{x^{2}}{x^{1}}\right)\right]-\alpha x^{1}, \\
& \mathcal{W}\left(x^{1}, x^{2}\right)=\frac{\lambda}{n}\left(x^{1} x^{1}+x^{2} x^{2}\right)^{\frac{n}{2}} \sin \left[n \arctan \left(\frac{x^{2}}{x^{1}}\right)\right]-\alpha x^{2},
\end{aligned}
$$

the one-parametric family

$$
\left(\begin{array}{l}
W^{(\alpha)}\left(x^{1}, x^{2}\right) \\
\mathcal{W}^{(\alpha)}\left(x^{1}, x^{2}\right)
\end{array}\right)=\left(\begin{array}{cc}
\cos \alpha & \sin \alpha \\
-\sin \alpha & \cos \alpha
\end{array}\right)\left(\begin{array}{l}
W\left(x^{1}, x^{2}\right) \\
\mathcal{W}\left(x^{1}, x^{2}\right)
\end{array}\right)
$$

forms such a circle of solutions. The proof is based on the fact that $W$ and $\mathcal{W}$ are harmonic conjugate functions and satisfy the real analytic Cauchy-Riemann equations $\frac{\partial W}{\partial x^{1}}=\frac{\partial \mathcal{W}}{\partial x^{2}}, \frac{\partial W}{\partial x^{2}}=-\frac{\partial \mathcal{W}}{\partial x^{1}}$, a necessary and sufficient condition to build $\mathcal{N}=4$ supersymmetric extensions in this system. 


\subsection{Quantum super Liouville Type I models}

There are other dynamical systems that are Hamilton-Jacobi separable in two dimensions. We shall focus on systems that are separable using elliptic coordinates classified by Liouville as Type I. For a thorough analysis of this kind of $\mathcal{N}=2$ supersymetric classical system, we refer to [12].

\subsubsection{Classical super Liouville models of Type I}

Let us consider the map $\xi: \mathbb{R}^{2} \longrightarrow \mathbb{D}^{2}$, where $\mathbb{D}^{2}$ is an open sub-set of $\mathbb{R}^{2}$, with coordinates $(u, v)$, and let $\xi^{-1}: \mathbb{D}^{2} \longrightarrow \mathbb{R}^{2}$ be the inverse map:

$$
\begin{gathered}
\left(x^{1}, x^{2}\right)=\xi^{-1}(u, v)=\left(\frac{1}{c} u v, \pm \frac{1}{c} \sqrt{\left(u^{2}-c^{2}\right)\left(c^{2}-v^{2}\right)}\right) \quad, \quad \xi\left(x^{1}, x^{2}\right)=(u, v) \\
u=\left(\frac{\sqrt{\left(x^{1}+c\right)^{2}+x^{2} x^{2}}+\sqrt{\left(x^{1}-c\right)^{2}+x^{2} x^{2}}}{2}\right), v=\left(\frac{\sqrt{\left(x^{1}+c\right)^{2}+x^{2} x^{2}}-\sqrt{\left(x^{1}-c\right)^{2}+x^{2} x^{2}}}{2}\right)
\end{gathered}
$$

The $u, v$ variables are the elliptic coordinates of the bosonic system: $u \in[c, \infty), v \in[-c, c]$ and $\mathbb{D}^{2}$ is the closure of the infinite strip: $\overline{\mathbb{D}}^{2}=[c, \infty) \times[-c, c]$. Let us assume the notation $\xi^{*}$ for the map induced in the functions on $\mathbb{R}^{2}$; i.e. $\xi^{*} U\left(x^{1}, x^{2}\right)=U\left(\xi\left(x^{1}, x^{2}\right)\right) \equiv U(u, v)$. Thus, we shall write $U$ for $U\left(x^{1}, x^{2}\right)$ and $\xi^{*} U$ for $U(u, v)$ and a similar convention will be used for the functions in the phase and co-phase spaces.

The Hamilton-Jacobi equation for zero energy and $V=-U$, formula (11), written in elliptic coordinates, reads:

$$
\xi^{*} U=\frac{u^{2}-c^{2}}{u^{2}-v^{2}} f(u)+\frac{c^{2}-v^{2}}{u^{2}-v^{2}} g(v)=\frac{1}{2} \frac{u^{2}-c^{2}}{u^{2}-v^{2}}\left(\frac{d F}{d u}\right)^{2}+\frac{1}{2} \frac{c^{2}-v^{2}}{u^{2}-v^{2}}\left(\frac{d G}{d v}\right)^{2},
$$

assuming separability: $\xi^{*} W=F(u)+G(v) \Rightarrow \frac{\partial^{2} \xi^{*} W}{\partial u \partial v}=0$. Note that $f(u), g(v)$ come from the bosonic potential. A complete solution of (12) consists of the four combinations of the two independent onedimensional problems:

$$
\begin{aligned}
F(u) & =\int d u \sqrt{2 f(u)} \quad, \quad G(v)=\int d v \sqrt{2 g(v)} \\
\xi^{*} W^{(a, b)} & =(-1)^{a} \int d u \sqrt{2 f(u)}+(-1)^{b} \int d v \sqrt{2 g(v)} \quad, a, b=0,1
\end{aligned}
$$

The map $\xi^{*}$ induces a non-Euclidean metric in $\mathbb{D}^{2}=(c, \infty) \times(-c, c)$ with metric tensor and Christoffel symbols:

$$
\begin{gathered}
g(u, v)=\left(\begin{array}{cc}
g_{u u}=\frac{u^{2}-v^{2}}{u^{2}-c^{2}} & g_{u v}=0 \\
g_{v u}=0 & g_{v v}=\frac{u^{2}-v^{2}}{c^{2}-v^{2}}
\end{array}\right) \quad, \quad g^{-1}(u, v)=\left(\begin{array}{cc}
g^{u u}=\frac{u^{2}-c^{2}}{u^{2}-v^{2}} & g^{u v}=0 \\
g^{v u}=0 & g^{v v}=\frac{c^{2}-v^{2}}{u^{2}-v^{2}}
\end{array}\right) \\
\Gamma_{u u}^{u}=\frac{-u\left(c^{2}-v^{2}\right)}{\left(u^{2}-v^{2}\right)\left(u^{2}-c^{2}\right)} \quad, \quad \Gamma_{v v}^{v}=\frac{v\left(u^{2}-c^{2}\right)}{\left(u^{2}-v^{2}\right)\left(c^{2}-v^{2}\right)} \quad, \quad \Gamma_{u v}^{u}=\Gamma_{v u}^{u}=\frac{-v}{u^{2}-v^{2}} \\
\Gamma_{u u}^{v}=\frac{v\left(c^{2}-v^{2}\right)}{\left(u^{2}-v^{2}\right)\left(u^{2}-c^{2}\right)} \quad, \quad \Gamma_{v v}^{u}=\frac{-u\left(u^{2}-c^{2}\right)}{\left(u^{2}-v^{2}\right)\left(c^{2}-v^{2}\right)} \quad, \quad \Gamma_{u v}^{v}=\Gamma_{v u}^{v}=\frac{u}{u^{2}-v^{2}}
\end{gathered}
$$

Besides the bosonic (even Grassman) variables $u, v$, there are also fermionic (odd Grassman) Majorana spinors $\vartheta_{\alpha}^{u}, \vartheta_{\alpha}^{v}$ in the system. We choose the zweibein

$$
g^{u u}(u, v)=\sum_{j=1}^{2} e_{j}^{u}(u, v) e_{j}^{u}(u, v) \quad, \quad g^{v v}(u, v)=\sum_{j=1}^{2} e_{j}^{v}(u, v) e_{j}^{v}(u, v)
$$


in the form:

$$
e_{1}^{u}(u, v)=\left(\frac{u^{2}-c^{2}}{u^{2}-v^{2}}\right)^{\frac{1}{2}} \quad, \quad e_{2}^{v}(u, v)=\left(\frac{c^{2}-v^{2}}{u^{2}-v^{2}}\right)^{\frac{1}{2}} .
$$

Curved and flat Grassman variables are related as: $\vartheta_{\alpha}^{u}(u, v)=e_{1}^{u}(u, v) \theta_{\alpha}^{1}, \vartheta_{\alpha}^{v}(u, v)=e_{1}^{v}(u, v) \theta_{\alpha}^{1}$.

A supersymmetric two-dimensional mechanical system is a super- Liouville model of Type I if the Lagrangian is of the form $\xi^{*} L=\xi^{*} L_{B}+\xi^{*} L_{F}+\xi^{*} L_{B F}$, with:

$$
\begin{aligned}
\xi^{*} L_{B}= & \frac{1}{2} g_{u u}(u, v) \dot{u} \dot{u}+\frac{1}{2} g_{v v}(u, v) \dot{v} \dot{v}-\frac{1}{2} g^{u u}(u, v)\left(\frac{d F}{d u}\right)^{2}-\frac{1}{2} g^{v v}(u, v)\left(\frac{d G}{d v}\right)^{2} \\
\xi^{*} L_{F}= & -\frac{i}{2} g_{u u}(u, v) \vartheta_{\alpha}^{u} D_{t} \vartheta_{\alpha}^{u}-\frac{i}{2} g_{v v}(u, v) \vartheta_{\alpha}^{v} D_{t} \vartheta_{\alpha}^{v} \\
\xi^{*} L_{B F}^{I}= & -i\left[\frac{d^{2} F}{d u^{2}}-\Gamma_{u u}^{u} \frac{d F}{d u}-\Gamma_{u u}^{v} \frac{d G}{d v}\right] \vartheta_{2}^{u} \vartheta_{1}^{u}-i\left[\frac{d^{2} G}{d v^{2}}-\Gamma_{v v}^{u} \frac{d F}{d u}-\Gamma_{v v}^{v} \frac{d G}{d v}\right] \vartheta_{2}^{v} \vartheta_{1}^{v}+ \\
& +i\left[\Gamma_{u v}^{u} \frac{d F}{d u}-\Gamma_{u v}^{v} \frac{d G}{d v}\right]\left(\vartheta_{2}^{v} \vartheta_{1}^{u}+\vartheta_{2}^{u} \vartheta_{1}^{v}\right)
\end{aligned}
$$

The fermionic kinetic energy is encoded in $\xi^{*} L_{F}$, where the covariant derivatives are defined as:

$$
\begin{aligned}
& D_{t} \vartheta_{\alpha}^{u}=\dot{\vartheta}_{\alpha}^{u}+\Gamma_{u u}^{u} \dot{u} \vartheta_{\alpha}^{u}+\Gamma_{u v}^{u} \dot{u} \vartheta_{\alpha}^{v}+\Gamma_{v u}^{u} \dot{v} \vartheta_{\alpha}^{u}+\Gamma_{v v}^{u} \dot{v} \vartheta_{\alpha}^{u} \\
& D_{t} \vartheta_{\alpha}^{v}=\dot{\vartheta}_{\alpha}^{v}+\Gamma_{u u}^{v} \dot{u} \vartheta_{\alpha}^{u}+\Gamma_{u v}^{v} \dot{u} \vartheta_{\alpha}^{v}+\Gamma_{v u}^{v} \dot{v} \vartheta_{\alpha}^{u}+\Gamma_{v v}^{v} \dot{v} \vartheta_{\alpha}^{v}
\end{aligned}
$$

The Yukawa terms governing the Bose-Fermi interactions are prescribed in $\xi^{*} L_{B F}$. The generalized momenta of the supersymmetric system and the supercharges are:

$$
\begin{aligned}
P_{u}= & g_{u u}(u, v)\left(\dot{u}-\frac{i}{2} \vartheta_{\alpha}^{u} \Gamma_{u v}^{u} \vartheta_{\alpha}^{v}\right)-\frac{i}{2} g_{v v}(u, v) \vartheta_{\alpha}^{v} \Gamma_{u u}^{v} \vartheta_{\alpha}^{u} \\
P_{v}= & g_{v v}(u, v)\left(\dot{v}-\frac{i}{2} \vartheta_{\alpha}^{v} \Gamma_{v u}^{v} \vartheta_{\alpha}^{u}\right)-\frac{i}{2} g_{u u}(u, v) \vartheta_{\alpha}^{u} \Gamma_{v v}^{u} \vartheta_{\alpha}^{v} \\
\xi^{*} Q_{1}^{(a, b)}= & P_{u} \vartheta_{1}^{u}+\frac{i}{2}\left(g_{u u} \vartheta_{\alpha}^{u} \Gamma_{u v}^{u} \vartheta_{\alpha}^{v}+g_{v v} \vartheta_{\alpha}^{v} \Gamma_{u u}^{v} \vartheta_{\alpha}^{u}\right) \vartheta_{1}^{u}-(-1)^{a} \frac{d F}{d u} \vartheta_{2}^{u} \\
& +P_{v} \vartheta_{1}^{v}+\frac{i}{2}\left(g_{v v} \vartheta_{\alpha}^{v} \Gamma_{v u}^{v} \vartheta_{\alpha}^{u}+g_{u u} \vartheta_{\alpha}^{u} \Gamma_{v v}^{u} \vartheta_{\alpha}^{v} \vartheta_{1}^{u}\right) \vartheta_{1}^{v}-(-1)^{b} \frac{d G}{d v} \vartheta_{2}^{v} \\
\xi^{*} Q_{2}^{(a, b)}= & P_{u} \vartheta_{2}^{u}+\frac{i}{2}\left(g_{u u} \vartheta_{\alpha}^{u} \Gamma_{u v}^{u} \vartheta_{\alpha}^{v}+g_{v v} \vartheta_{\alpha}^{v} \Gamma_{u u}^{v} \vartheta_{\alpha}^{u}\right) \vartheta_{2}^{u}+(-1)^{a} \frac{d F}{d u} \vartheta_{1}^{u} \\
& +P_{v} \vartheta_{2}^{v}+\frac{i}{2}\left(g_{v v} \vartheta_{\alpha}^{v} \Gamma_{v u}^{v} \vartheta_{\alpha}^{u}+g_{u u} \vartheta_{\alpha}^{u} \Gamma_{v v}^{u} \vartheta_{\alpha}^{v} \vartheta_{1}^{u}\right) \vartheta_{2}^{u}+(-1)^{b} \frac{d G}{d v} \vartheta_{1}^{v}
\end{aligned}
$$

\subsubsection{Quantum supercharges and Hamiltonian}

Passing to Majorana-Weyl spinors, $\vartheta_{+}^{u, v}=\frac{1}{\sqrt{2}}\left(\vartheta_{2}^{u, v}-i \vartheta_{1}^{u, v}\right), \vartheta_{-}^{u, v}=-\frac{1}{\sqrt{2}}\left(\vartheta_{2}^{u, v}+i \vartheta_{1}^{u, v}\right)$, the fermionic quantization rules lead us to the Fermi operators in non-Euclidean space: $\psi_{ \pm}^{u}(u, v)=e_{1}^{u}(u, v) \psi_{ \pm}^{1}, \psi_{ \pm}^{v}(u, v)=$ $e_{2}^{v}(u, v) \psi_{ \pm}^{2}$. Setting e.g. $a=b=0$, and also quantizing the generalized momenta, $\hat{P}_{u}=\frac{1}{i} \frac{\partial}{\partial u}, \hat{P}_{v}=\frac{1}{i} \frac{\partial}{\partial v}$, we obtain the quantum supercharges:

$$
\xi^{*} \hat{Q}_{ \pm}=-i \psi_{ \pm}^{u} \nabla_{u}^{\mp}-i \frac{u}{c^{2}-v^{2}} \psi_{ \pm}^{u} \psi_{ \pm}^{v} \psi_{\mp}^{v}-i \psi_{ \pm}^{v} \nabla_{v}^{\mp}+i \frac{v}{u^{2}-c^{2}} \psi_{ \pm}^{v} \psi_{ \pm}^{u} \psi_{\mp}^{u} \quad
$$

or, in matrix form:

$$
\xi^{*} \hat{Q}_{+}=-i\left(\begin{array}{cccc}
0 & 0 & 0 & 0 \\
e_{1}^{u} \nabla_{u}^{-} & 0 & 0 & 0 \\
e_{2}^{v} \nabla_{v}^{-} & 0 & 0 & 0 \\
0 & -e_{2}^{v}\left(\nabla_{v}^{-}-\frac{v}{u^{2}-v^{2}}\right) & e_{1}^{u}\left(\nabla_{u}^{-}+\frac{u}{u^{2}-v^{2}}\right) & 0
\end{array}\right) \quad, \quad \nabla_{u}^{\mp}=\frac{\partial}{\partial u} \mp \frac{d F}{d u}
$$




$$
\xi^{*} \hat{Q}_{-}=-i\left(\begin{array}{cccc}
0 & e_{1}^{u}\left(\nabla_{u}^{+}+\frac{u}{u^{2}-v^{2}}\right) & e_{2}^{v}\left(\nabla_{v}^{+}-\frac{v}{u^{2}-v^{2}}\right) & 0 \\
0 & 0 & 0 & -e_{2}^{v} \nabla_{v}^{+} \\
0 & 0 & 0 & e_{1}^{u} \nabla_{u}^{+} \\
0 & 0 & 0 & 0
\end{array}\right) \quad, \quad \nabla_{v}^{\mp}=\frac{\partial}{\partial v} \mp \frac{d G}{d v}
$$

In order to make clear how separability and supersymmetry are entangled, it is convenient to write the different pieces of the quantum Hamiltonian, $\xi^{*} \hat{H}=\frac{1}{2}\left\{\xi^{*} \hat{Q}_{+}, \xi^{*} \hat{Q}_{-}\right\}$,

$$
\xi^{*} \hat{H}=\frac{1}{2\left(u^{2}-v^{2}\right)}\left(\begin{array}{ccc}
\xi^{*} \hat{h}^{(0)}\left(\frac{\partial}{\partial u}, \frac{\partial}{\partial v}, u, v\right) & 0 & 0 \\
0 & \xi^{*} \hat{h}^{(1)}\left(\frac{\partial}{\partial u}, \frac{\partial}{\partial v}, u, v\right) & 0 \\
0 & 0 & \xi^{*} \hat{h}^{(2)}\left(\frac{\partial}{\partial u}, \frac{\partial}{\partial v}, u, v\right)
\end{array}\right)
$$

separately. On the subspaces $\mathcal{H}_{0}$ and $\mathcal{H}_{2}$, the differential operator $\xi^{*} \hat{H}$ splits into the following structure:

- $\xi^{*} \hat{h}^{(0)}\left(\frac{\partial}{\partial u}, \frac{\partial}{\partial v}, u, v\right)=\hat{j}^{(0)}\left(\frac{\partial}{\partial u}, u\right)+\hat{k}^{(0)}\left(\frac{\partial}{\partial v}, v\right)$.

$$
\begin{aligned}
& \hat{j}^{(0)}\left(\frac{\partial}{\partial u}, u\right)=\left(u^{2}-c^{2}\right)\left[-\frac{\partial^{2}}{\partial u^{2}}-\frac{u}{u^{2}-c^{2}} \frac{\partial}{\partial u}+\left(\frac{d F}{d u}\right)^{2}+\frac{d^{2} F}{d u^{2}}+\frac{u}{u^{2}-c^{2}} \frac{d F}{d u}\right] \\
& \hat{k}^{(0)}\left(\frac{\partial}{\partial v}, v\right)=\left(c^{2}-v^{2}\right)\left[-\frac{\partial^{2}}{\partial v^{2}}+\frac{v}{c^{2}-v^{2}} \frac{\partial}{\partial v}+\left(\frac{d G}{d v}\right)^{2}+\frac{d^{2} G}{d v^{2}}-\frac{v}{c^{2}-v^{2}} \frac{d G}{d v}\right]
\end{aligned}
$$

- $\xi^{*} \hat{h}^{(2)}\left(\frac{\partial}{\partial u}, \frac{\partial}{\partial v}, u, v\right)=\hat{j}^{(2)}\left(\frac{\partial}{\partial u}, u\right)+\hat{k}^{(2)}\left(\frac{\partial}{\partial v}, v\right)$.

$$
\begin{gathered}
\hat{j}^{(2)}\left(\frac{\partial}{\partial u}, u\right)=\left(u^{2}-c^{2}\right)\left[-\frac{\partial^{2}}{\partial u^{2}}-\frac{u}{u^{2}-c^{2}} \frac{\partial}{\partial u}+\left(\frac{d F}{d u}\right)^{2}-\frac{d^{2} F}{d u^{2}}-\frac{u}{u^{2}-c^{2}} \frac{d F}{d u}\right] \\
\hat{k}^{(2)}\left(\frac{\partial}{\partial v}, v\right)=\left(c^{2}-v^{2}\right)\left[-\frac{\partial^{2}}{\partial v^{2}}+\frac{v}{c^{2}-v^{2}} \frac{\partial}{\partial v}+\left(\frac{d G}{d v}\right)^{2}-\frac{d^{2} G}{d v^{2}}+\frac{v}{c^{2}-v^{2}} \frac{d G}{d v}\right] .
\end{gathered}
$$

Therefore, we conclude that in the bosonic sectors the dynamical problem is separable in the $u$ and $v$ variables.

Things, however, become more involved in the fermionic sectors. We write the Hamiltonian acting on $\mathcal{H}_{1}$ as follows:

$$
\xi^{*} \hat{h}^{(1)}\left(\frac{\partial}{\partial u}, \frac{\partial}{\partial v}, u, v\right)=\left(\begin{array}{cc}
l_{+}^{(1)}\left(\frac{\partial}{\partial u}, u\right)+f_{+}^{(1)}\left(\frac{\partial}{\partial v}, v\right)+g_{+}^{(1)}(u, v) & t_{+}^{(1)}(u, v) \\
t_{-}^{(1)}(u, v) & l_{-}^{(1)}\left(\frac{\partial}{\partial u}, u\right)+f_{-}^{(1)}\left(\frac{\partial}{\partial v}, v\right)+g_{-}^{(1)}(u, v)
\end{array}\right)
$$

Here,

$$
\begin{gathered}
l_{ \pm}^{(1)}\left(\frac{\partial}{\partial u}, u\right)=\left(u^{2}-c^{2}\right)\left[-\frac{\partial^{2}}{\partial u^{2}}-\frac{u}{u^{2}-c^{2}} \frac{\partial}{\partial u}+\left(\frac{d F}{d u}\right)^{2} \mp \frac{d^{2} F}{d u^{2}}\right], \\
f_{ \pm}^{(1)}\left(\frac{\partial}{\partial u}, u\right)=\left(c^{2}-v^{2}\right)\left[-\frac{\partial^{2}}{\partial v^{2}}+\frac{v}{c^{2}-v^{2}} \frac{\partial}{\partial v}+\left(\frac{d G}{d v}\right)^{2} \pm \frac{d^{2} G}{d v^{2}}\right], \\
g_{ \pm}^{(1)}(u, v)=\frac{\left(u^{2}+v^{2}-2 c^{2}\right)}{u^{2}-v^{2}}\left[ \pm u \frac{d F}{d u} \mp v \frac{d G}{d v}\right] \quad, \quad t_{ \pm}^{(1)}(u, v)=\frac{\sqrt{\left(u^{2}-c^{2}\right)\left(c^{2}-v^{2}\right)}}{\left(u^{2}-v^{2}\right)^{2}}\left(v \frac{d F}{d u}+u \frac{d G}{d v}\right)
\end{gathered}
$$

The variables $u$ and $v$ are mixed in $\xi^{*} \hat{h}^{(1)}$. It seems that supersymmetry breaks down separability. Nevertheless, the non-null spectrum of $\xi^{*} \hat{h}^{(1)}$ is given by the non-null spectra of $\xi^{*} \hat{h}^{(0)}$ and $\xi^{*} \hat{h}^{(2)}$, operators with separable spectral problems. 


\section{Two examples in two dimensions}

\subsection{The Planar anisotropic harmonic oscillator}

This is a Type IV Liouville model. If $a_{1}, a_{2}=0,1$, the potential, superpotentials and supercharges are:

$$
\begin{aligned}
& U\left(x_{1}, x_{2}\right)=\frac{k_{1}}{2} x_{1}^{2}+\frac{k_{2}}{2} x_{2}^{2} \quad, \quad W^{\left(a_{1}, a_{2}\right)}\left(x_{1}, x_{2}\right)=\frac{(-1)^{a_{1}}}{2} \sqrt{k_{1} m_{1}} x_{1}^{2}+\frac{(-1)^{a_{2}}}{2} \sqrt{k_{2} m_{2}} x_{2}^{2} \\
& \hat{Q}_{+}^{\left(a_{1}, a_{2}\right)}=i \sqrt{2}\left(\begin{array}{cccc}
0 & 0 & 0 & 0 \\
\hat{q}_{1}^{\left(a_{1}\right)} & 0 & 0 & 0 \\
\hat{q}_{2}^{\left(a_{2}\right)} & 0 & 0 & 0 \\
0 & -\hat{q}_{2}^{\left(a_{2}\right)} & \hat{q}_{1}^{\left(a_{1}\right)} & 0
\end{array}\right) ; \hat{Q}_{-}^{\left(a_{1}, a_{2}\right)}=i \sqrt{2}\left(\begin{array}{cccc}
0 & \hat{q}_{1}^{\left(a_{1}\right)^{\dagger}} & \hat{q}_{2}^{\left(a_{2}\right)^{\dagger}} & 0 \\
0 & 0 & 0 & -\hat{q}_{2}^{\left(a_{2}\right)^{\dagger}} \\
0 & 0 & 0 & \hat{q}_{1}^{\left(a_{1}\right)^{\dagger}} \\
0 & 0 & 0 & 0
\end{array}\right) \\
& \hat{q}_{1}^{\left(a_{1}\right)}=\frac{1}{\sqrt{2}} \frac{\partial}{\partial x_{1}}-(-1)^{a_{1}} \sqrt{\frac{k_{1} m_{1}}{2}} x_{1} \quad, \quad \hat{q}_{2}^{\left(a_{2}\right)}=\frac{1}{\sqrt{2}} \frac{\partial}{\partial x_{2}}-(-1)^{a_{2}} \sqrt{\frac{k_{2} m_{2}}{2}} x_{2} .
\end{aligned}
$$

From the annihilation operators

$$
\hat{A}_{1}=\frac{1}{\sqrt{2 m_{1}}} \frac{\partial}{\partial x_{1}}+\sqrt{\frac{k_{1}}{2}} x_{1} \quad, \quad \hat{A}_{2}=\frac{1}{\sqrt{2 m_{2}}} \frac{\partial}{\partial x_{2}}+\sqrt{\frac{k_{2}}{2}} x_{2} \quad,
$$

their adjoints, and the natural frequencies $\omega_{1}=\sqrt{\frac{k_{1}}{m_{1}}}, \omega_{2}=\sqrt{\frac{k_{2}}{m_{2}}}$ one obtains the Hamiltonian:

$$
\begin{aligned}
& \hat{h}^{(0)}=\sum_{j=1}^{2} \omega_{j}\left(\hat{A}_{j}^{\dagger} \hat{A}_{j}+\frac{1}{2}\left(1+(-1)^{a_{j}}\right)\right) \quad, \quad \hat{h}^{(2)}=\sum_{j=1}^{2} \omega_{j}\left(\hat{A}_{j}^{\dagger} \hat{A}_{j}+\frac{1}{2}\left(1-(-1)^{a_{j}}\right)\right), \\
& \hat{h}^{(1)}=\left(\begin{array}{cc}
\sum_{j=1}^{2} \omega_{j}\left(\hat{A}_{j}^{\dagger} \hat{A}_{j}+\frac{1}{2}\right)-\frac{(-1)^{a_{1}}}{2} \omega_{1}+\frac{(-1)^{a_{2}}}{2} \omega_{2} & 0 \\
0 & \sum_{j=1}^{2} \omega_{j}\left(\hat{A}_{j}^{\dagger} \hat{A}_{j}+\frac{1}{2}\right)+\frac{(-1)^{a_{1}}}{2} \omega_{1}-\frac{(-1)^{a_{2}}}{2} \omega_{2}
\end{array}\right) .
\end{aligned}
$$

The Fock space basis

$$
\hat{A}_{1}|0,0\rangle=\hat{A}_{2}|0,0\rangle=0 \quad, \quad\left|n_{1}, n_{2}\right\rangle=\frac{1}{\sqrt{n_{1} ! n_{2} !}}\left(\hat{A}_{1}^{\dagger}\right)^{n_{1}}\left(\hat{A}_{2}^{\dagger}\right)^{n_{2}}|0,0\rangle
$$

provides the eigenfunctions: $\hat{A}_{1}^{\dagger} \hat{A}_{1}\left|n_{1}, n_{2}\right\rangle=n_{1}\left|n_{1}, n_{2}\right\rangle, \hat{A}_{2}^{\dagger} \hat{A}_{2}\left|n_{1}, n_{2}\right\rangle=n_{2}\left|n_{1}, n_{2}\right\rangle$. Thus,

$$
\operatorname{Spec} \hat{h}^{(0)}=\sqcup_{j=1}^{2} \omega_{j}\left[n_{j}+\frac{1}{2}\left(1+(-1)^{a_{j}}\right)\right] \quad, \quad \operatorname{Spec} \hat{h}^{(2)}=\sqcup_{j=1}^{2} \omega_{j}\left[n_{j}+\frac{1}{2}\left(1-(-1)^{a_{j}}\right)\right]
$$

$$
\text { Spec } \hat{h}^{(1)}=\sqcup_{j=1}^{2} \omega_{j}\left(n_{j}+\frac{1}{2}\right) \mp \frac{(-1)^{a_{1}}}{2} \omega_{1} \pm \frac{(-1)^{a_{2}}}{2} \omega_{2} \quad .
$$

The ground state

$$
\Psi\left(x_{1}, x_{2}\right)=\left\langle x_{1}, x_{2} \mid 0,0\right\rangle=\exp \left[-\frac{1}{2} \sum_{j=1}^{2} \omega_{j} m_{j} x_{j}^{2}\right]
$$

belongs to: (a) $\mathcal{H}_{0}$, if $a_{1}=a_{2}=1$, (b) $\mathcal{H}_{2}$, if $a_{1}=a_{2}=0$, (c) $\mathcal{H}_{1}$, if $a_{1} \neq a_{2}$. For the $a_{1}=a_{2}=1$ case, the SUSY partner states - all of them with energy $E=n_{1} \omega_{1}+n_{2} \omega_{2}$ - are:

$$
\begin{aligned}
& \left(\begin{array}{c}
\left|n_{1}, n_{2}\right\rangle \\
0 \\
0 \\
0
\end{array}\right)
\end{aligned}
$$

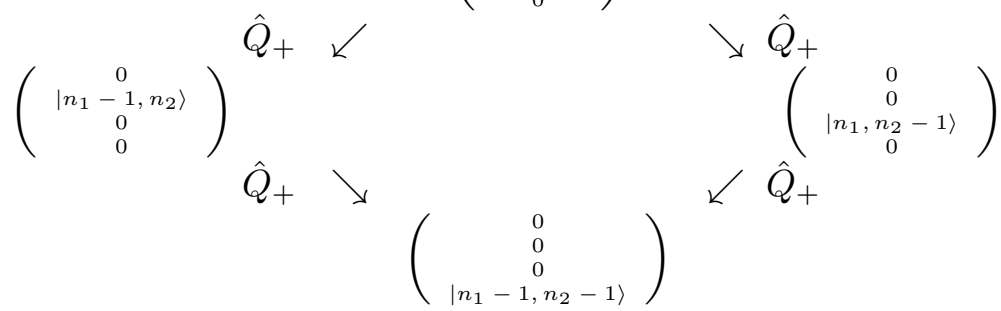

Other choices for $a_{1}$ and $a_{2}$ require permutations between the vertices of the rhombus. 


\subsection{Two Newtonian centers of force on a plane}

Let us start with the energy potential for the problem of two attractive centers of force with non-equal strengths (see Figure 1(a)):

$$
U\left(x_{1}, x_{2}\right)=-\left(\frac{\alpha_{1}}{r_{1}}+\frac{\alpha_{2}}{r_{2}}\right) \quad, \quad 0<\alpha_{2}<\alpha_{1} \quad,
$$
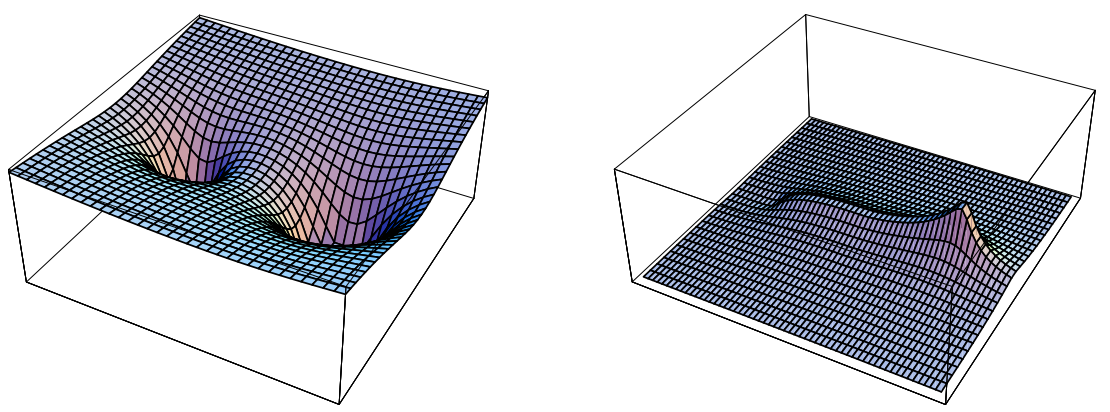

Figure 1: a) $U\left(x_{1}, x_{2}\right), c=1, \alpha_{1}=2, \alpha_{2}=1$. b) $\exp \left(W\left(x_{1}, x_{2}\right)\right)$.

The distances from the centers are appropriately given in terms of the elliptic coordinates: $u=$ $\frac{1}{2}\left(r_{1}+r_{2}\right), v=\frac{1}{2}\left(r_{2}-r_{1}\right), r_{1}=\sqrt{\left(x_{1}-c\right)^{2}+x_{2}^{2}}, r_{2}=\sqrt{\left(x_{1}+c\right)^{2}+x_{2}^{2}}$. The Hamiltonian in elliptic coordinates,

$$
\xi^{*} H=\frac{1}{2} \frac{1}{u^{2}-v^{2}}\left[\left(u^{2}-c^{2}\right) p_{u}^{2}+\left(c^{2}-v^{2}\right) p_{v}^{2}\right]+\frac{k_{+} u-k_{-} v}{u^{2}-v^{2}} \quad,
$$

depends on the coupling constants $k_{ \pm}=\alpha_{2} \pm \alpha_{1}$ and shows that we are dealing with a type I Liouville system. The ansatz $S=-i_{1} t+F\left[u ; i_{1}, i_{2}\right]+G\left[v ; i_{1}, i_{2}\right]$ leads to the $i_{1}=i_{2}=0$ Hamilton-Jacobi equation:

$$
\frac{k_{+} u-k_{-} v}{u^{2}-v^{2}}=\frac{1}{2} g^{u u}\left(\frac{d F}{d u}\right)^{2}+\frac{1}{2} g^{v v}\left(\frac{d G}{d v}\right)^{2} \quad \text {. }
$$

Note that in this case the potential energy is semi-definite negative and, to find real solutions, we do not replace $U$ by $-U$ in the Hamilton-Jacobi equation. Therefore, the solution of (17) in terms of the elliptic and complete elliptic integrals of the first and second kind, [18],

$$
\begin{aligned}
& F(u)=-2 \sqrt{k_{+} c}\left[\mathrm{~F}\left(\sin ^{-1} \sqrt{\frac{u-c}{u}}, \frac{1}{2}\right)-2 \mathrm{E}\left(\sin ^{-1} \sqrt{\frac{u-c}{u}}, \frac{1}{2}\right)+\sqrt{\frac{2\left(u^{2}-c^{2}\right)}{u c}}\right], \\
& G(v)=\left\{\begin{array}{cc}
2 \sqrt{k_{-} c}\left[2 \mathrm{E}\left(\sin ^{-1} \sqrt{\frac{2 v}{v-c}}, \frac{1}{2}\right)-\mathrm{F}\left(\sin ^{-1} \sqrt{\frac{2 v}{v-c}}, \frac{1}{2}\right)-\sqrt{\frac{2 v(v+c)}{c(v-c)}}\right] & -c<v \leq 0 \\
2 i \sqrt{k_{-} c}\left[2 \mathrm{E}\left(\sin ^{-1} \sqrt{\frac{c-v}{c}}, \frac{1}{2}\right)-2 \mathrm{E}[1 / 2]+\mathrm{F}\left(\sin ^{-1} \sqrt{\frac{c-v}{c}}, \frac{1}{2}\right)-\mathrm{K}[1 / 2]\right] & 0 \leq v<c
\end{array}\right.
\end{aligned}
$$

provide the superpotentials

$$
\xi^{*} W^{(a, b)}\left(x_{1}, x_{2}\right)=(-1)^{a} F(u)+(-1)^{b} G(v)
$$

for two repulsive Newtonian centers. Nevertheless, the Laplacian of the superpotential - given by the terms

$$
\begin{array}{ccc}
\frac{d F}{d u}=-\sqrt{\frac{2 k_{+} u}{u^{2}-c^{2}}} & , & \frac{d G}{d v}=-\sqrt{\frac{-2 k_{-} v}{c^{2}-v^{2}}} \\
\frac{d^{2} F}{d u^{2}}=-\frac{1}{2} \frac{u^{2}+c^{2}}{u\left(u^{2}-c^{2}\right)} \frac{d F}{d u} & , & \frac{d^{2} G}{d v^{2}}=\frac{1}{2} \frac{c^{2}+v^{2}}{v\left(c^{2}-v^{2}\right)} \frac{d G}{d v}
\end{array}
$$


coming from the quantization of the Yukawa couplings- induce attractive forces in the supersymmetric extension of two repulsive Newtonian centers and there is hope of finding normalizable eigenstates.

In fact, choosing $a=b=0$ we obtain the zero-energy wave function in the Bose/Bose sector, $\hat{Q}_{+} \Psi_{0}\left(x_{1}, x_{2}\right)=0$ :

$$
i\left(\begin{array}{cccc}
0 & 0 & 0 & 0 \\
e_{1}^{u}\left(\frac{\partial}{\partial u}-\frac{d F}{d u}\right) & 0 & 0 & 0 \\
e_{2}^{v}\left(\frac{\partial}{\partial v}-\frac{d G}{d v}\right) & 0 & 0 & 0 \\
0 & -e_{2}^{v}\left(\frac{\partial}{\partial v}-\frac{d G}{d v}-\frac{v}{u^{2}-v^{2}}\right) & e_{1}^{u}\left(\frac{\partial}{\partial u}-\frac{d F}{d u}+\frac{u}{u^{2}-v^{2}}\right) & 0
\end{array}\right)\left(\begin{array}{c}
\psi_{0}(u, v) \\
0 \\
0 \\
0
\end{array}\right)=0
$$

if

Therefore,

$$
e_{1}^{u} \frac{\partial \log \psi_{0}}{\partial u}(u, v) \vec{e}_{1}+e_{2}^{v} \frac{\partial \log \psi_{0}}{\partial v}(u, v) \vec{e}_{2}=e_{1}^{u} \frac{d F}{d u} \vec{e}_{1}+e_{2}^{v} \frac{d G}{d v} \vec{e}_{2}
$$

$$
\psi_{0}(u, v)=\xi^{*} \Psi_{0}^{(0)}\left(x_{1}, x_{2}\right)=C \exp [F(u)+G(v)] \quad,
$$

which is normalizable, see Figure 2, is the ground state of the $\mathcal{N}=2$ supersymmetric particle, even though the particle's "body" is repelled by two centers.
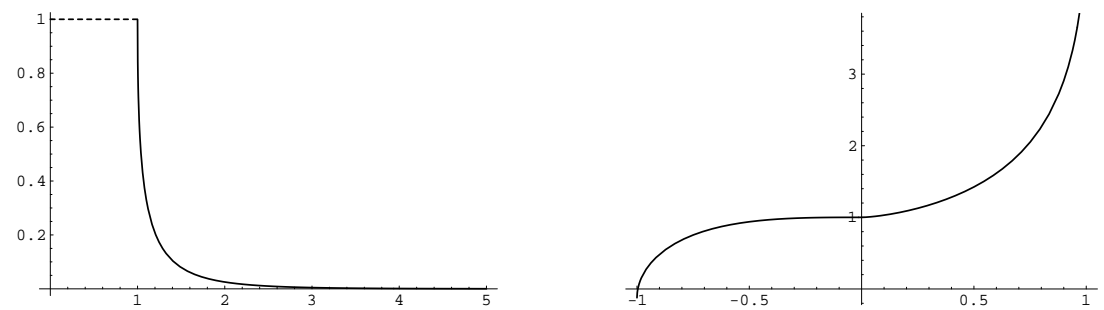

Figure 2: Plot of $\exp [F(u)]$ and $\exp [G(v)]$ as a function of $\mathrm{u}$ and $\mathrm{v}$ respectively.

Figure 1(b) shows a plot of the (18) wave function in Cartesian coordinates. It is amusing to check how well it fits in with the expected behaviour of a quantum particle in a potential well with two Newtonian holes; see, e.g. , [19] to find an approximate wave function for the ground state of the molecule-ion of hydrogen. The reason is that the effective quantum potential in the $\mathcal{H}_{0}$ sub-space

$$
\begin{gathered}
\hat{V}^{(0)}\left(x_{1}, x_{2}\right)=\frac{1}{2}\left(\Psi_{0}^{(0)}\right)^{-1}\left(x_{1}, x_{2}\right) \nabla^{2} \Psi_{0}^{(0)}\left(x_{1}, x_{2}\right) \\
\xi^{*} \hat{V}^{(0)}\left(x_{1}, x_{2}\right)=\frac{1}{2} \frac{\psi_{0}^{-1}(u, v)}{u^{2}-v^{2}}\left\{\left(u^{2}-c^{2}\right)\left(\frac{\partial}{\partial u}+\frac{u}{u^{2}-c^{2}}\right) \frac{\partial \psi_{0}}{\partial u}(u, v)+\left(c^{2}-v^{2}\right)\left(\frac{\partial}{\partial v}-\frac{v}{c^{2}-v^{2}}\right) \frac{\partial \psi_{0}}{\partial v}(u, v)\right\}
\end{gathered}
$$

is attractive towards the two centers.

\section{Summary}

Interactions in supersymmetric classical or quantum mechanics are prescribed by superpotentials. In this paper we have dealt with the following inverse problem: Given a Hamiltonian system, is there a superpotential from which forces are derived? If so, a supersymetric extension of this particular physical system is possible. We have encountered a two-fold way to meeting ambiguities in answering this question.

1. First, the outcome depends on the framework. For classical systems, superpotentials are solutions of zero-energy time-independent Hamilton-Jacobi equations. In the quantum domain superpotentials solve Ricatti-like PDE's. Moreover, canonical quantization and supersymmetric extension do not commute: the supersymmetric extension of - e.g., the quantum Coulomb problem- differs fom the quantization of the classical supersymmetric Coulomb system. 
2. In dimensions higher than one, superpotentials are far from unique. For instance, in HamiltonJacobi separable systems there are $2^{N}$ different superpotentials leading to supersymmetric systems with the same "body" dynamics. The ground states can be easily found in this kind of system because one needs to solve only first-order ODE's: one per each variable in which the dynamics separates.

\section{References}

[1] E. Witten, Nucl. Phys. B188(1981) 513 and Nucl. Phys. B202 (1982) 253

[2] E. Witten, J. Diff. Geom. 17 (1982) 661

[3] R. Casalbuoni, Nuovo Cim. A33 (1976)389

[4] F. A. Berezin and M. Marinov, Ann. Phys. 104 (1977) 336

[5] F. Cooper, A. Khare and U. Sukhatme, Phys. Rep.. 25 (1995)268

[6] G. Junker, Supersymmetric methods in quantum and statistical physics, Springer, Berlin, 1996

[7] A. Andrianov, N. Borisov and M. Ioffe, Phys. Lett A105 (1984) 19 and Theor. Math. Phys. 61 (1984) 1078

[8] A. Andrianov, N. Borisov. M. Eides and M. Ioffe, Phys. Lett A109 (1985) 143 and Theor. Math. Phys. 61 (1984) 963

[9] A. Andrianov, M. Ioffe and D. Nishnianidze, Jour. Phys. A32 (1999) 4641

[10] A. Andrianov, M. Ioffe and D. Nishnianidze, arXiv: solv-int/9605007

[11] F. Cannata, M. Ioffe and D. Nishnianidze, Jour. Phys. A35 (2002) 1389, Phys. Lett. A310 (2003) 344

[12] A. Alonso-Izquierdo, M.A. Gonzalez Leon, J. Mateos Guilarte and M. de la Torre Mayado, Ann. Phys. 308 (2003)664

[13] A. Kirchberg, J. Lange, P. Pisani, A. Wipf, Ann. Phys. 303 (2003) 359

[14] R. Heumann, Jour. Phys. A35 (2002) 7437

[15] S. Cecotti and L. Girardello, Phys. Lett. B119 (1982)39

[16] J. Liouville, J. Math. Phys. Appl. 11 (1849) 345. A. Perelomov, Integrable Systems of Classical Mechanics and Lie Algebras, Birkhauser, (1992).

[17] A. Alonso-Izquierdo, M.A. Gonzalez Leon and J. Mateos Guilarte, Phys. Lett. B480 (2000)373

[18] M. Abramowitz and I. Stegun, Handbook of Mathematical Functions, Dover, New York

[19] L. Pauling and B. Wilson, Introduction to Quantum Mechanics, Dover, New York 